HNO 2010 $\cdot 58: 324$

DOI 10.1007/s00106-010-2093-y

Online publiziert: 26. März 2010

(c) Springer-Verlag 2010

\author{
K. Hörmann \\ Universitäts-Hals-Nasen-Ohren-Klinik, Medizinische Fakultät Mannheim \\ der Ruprecht-Karls-Universität Heidelberg, Mannheim
}

\title{
Endoskopie: Herausforderungen in der HNO-Heilkunde
}

Für die HNO-Heilkunde ergeben sich im interdisziplinär befruchtenden, aber auch kompetitiven Umfeld der Endoskopie aus meiner Sicht 2 Schwerpunkte: - einerseits die konsequente und flächendeckende Etablierung der endoskopischen Routine mit starren und flexiblen Instrumenten;

- andererseits das Vorantreiben neuer endoskopischer Arbeitsfelder.

Nur so können wir eine breite endoskopische Expertise für die HNO-Heilkunde erhalten und weiterentwickeln.

Ein Beispiel ist die perkutan-endoskopische Gastrostomie, welche mittlerweile zur Routine vor Einleitung einer Radiochemotherapie im Kopf-Hals-Bereich gehört. In den HNO-Kliniken, die nicht über flexible Instrumente verfügen, kann der Eingriff nicht von der HNO durchgeführt werden. Ist die Expertise vorhanden, legen die HNO-Ärzte die PEG selbst, was für die Langzeitbetreuung der Tumorpatienten von Vorteil ist (s. Beitrag von Mantsopoulos et al. in diesem Heft).

Die Erschließung neuer Tätigkeitsfelder umfasst beispielsweise die Miniendoskopie kleiner Kanäle (s. Beitrag von Koch et al. in der vorliegenden Ausgabe), bei der wir je nach Lokalisation noch völlig am Anfang (Tränenwege) bzw. an der Schwelle zu ihrer Etablierung (Speichelgänge) stehen. Hier bietet sich erneut die Chance, bisher transkutan geführte Operationen zum Wohle unserer Patienten durch minimal-invasive, endoskopische Verfahren zu ersetzen. In der Schlafmedizin - ein ebenfalls interdisziplinäres Fach - sind die Erfolge der chirurgischen Be- handlung der Schlafapnoe nach wie vor unbefriedigend, was uns berechtigter Kritik der Nachbardisziplinen aussetzt. Die Videoendoskopie des Pharynx in Sedierung (s. Beitrag von Maurer u. Hörmann in dieser Ausgabe) stellt zwar keine vollständig neue Technik per se, jedoch einen neuen endoskopischen Untersuchungsgang dar. Eine verbesserte Patientenselektion und damit eine verbesserte Erfolgsrate der Chirurgie bei obstruktiver Schlafapnoe ist das Ziel der aktuellen Forschung.

\section{Endoskopie und HNO gehören untrennbar zusammen}

Endoskopie und HNO gehören untrennbar zusammen. Dies Heft soll dazu beitragen, dass dieser Satz auch für die junge Generation von HNO-Ärzten so bleibt.

Viel Freude an der Lektüre wünscht Ihnen

Ihr

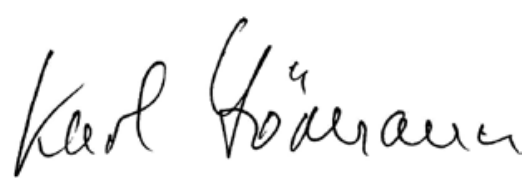

Karl Hörmann

\section{Korrespondenzadresse \\ Prof. Dr. K. Hörmann}

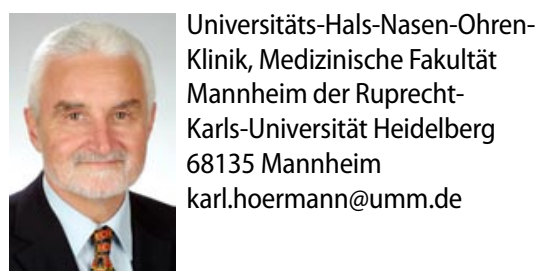

